\title{
A Study of Digitalization in HRM and its Effectiveness in Execution of HR Strategies and Policies
}

\author{
${ }^{* 1}$ Mruthyanjaya Rao Mangipudi, ${ }^{2}$ Rajesh Vaidya \\ ${ }^{1}$ Manager, Karvy Stock Broking Limited, Hyderabad and Research Scholar, RTM Nagpur University \\ ${ }^{2}$ Assistant Professor, Department of Management Technology, Shri Ramdeobaba College of Engineering and \\ Management, Ramdeo Tekdi, Gittikhadan, Katol Road, Nagpur - 444013 (Maharashtra State), India \\ Email:raomangipudi@gmail.com,rwvaidya@gmail.com
}

Received: $20^{\text {th }}$ September 2018, Accepted: $11^{\text {th }}$ October 2018, Published: $3^{\text {st }}$ October 2018

\begin{abstract}
This research paper is to investigate the emerging trends of human resource management with reference to digitalization in HRM. The paper will discuss the evolution of digitalization in HRM over a period of time and its contemporary application in execution of HRM strategies and policies. One of the foremost areas the HR should focus upon is "execution". The problem statement revolves around "execution", though the organization has a solid vision, excellent strategy and right talent. The top priority for the HR is to translate this strategy and vision into execution and make it a huge success. It is very pertinent to ensure the workforce in an organization is aligned to its strategy and priority. Especially, growing organizations do not have the luxury of having exclusive people assigned to do exclusive jobs. It is always all hands on deck scenario all the time. It is wise to note that the organizations are judged not only on their financial health, quality of service or workforce satisfaction, but also on how effectively they integrate with the external world, customers, partners and society at large. For this, the HR Managers have adopted the new ways of discharging their functions using innovate methods and tools like - Cloud HR technology, Artificial Intelligent driven tools, Adjusting the existing operating models, Adding strategic value to the businesses, Automation of mission critical HR functions such as a) talent acquisition, talent evaluation, c) on boarding, d) performance management, e) compensation \& benefits, f) maximizing the economies of scale, which means executing it better, faster and cheaper.
\end{abstract}

Keywords

Digitalization, Execution, Cloud HR Technology, Automation of HRM

\section{Introduction}

The nature of HR has evolved to a strategic function of an organization. The role of HR leaders in attracting, developing and enabling talent is critical to the success of any business. For this, it was inevitable for the HRM (Human Resources Management) to get transformed into Digital, which radically altered the way the employees and the businesses work. Digital Technology has entirely reinvented and enhanced the processes and systems in various functions across the industries, and the HR function is no exception. In the last two decades, digital tools like online job boards, applicant tracking systems (ATS), professional networking sites viz. LinkedIn, Facebook, Twitter have significantly changed the HR-dispensation right from a job posting or job application to recruitment and onboarding, employee engagement, performance management, and finally employee separation - capturing valuable insights during exit interview.HR started replacing manual processes which are of repetitive in nature, with automations. The need is to connect all HR processes across the organization over a period of time for greater transparency and efficiency. However, digitalization of HRM should not simply be looked at only a makeover process. HR leaders must be sensitive to the fact that it entails a huge change, and employees must be hand-held throughout this change failing which it is like a broken chain. Let us not forget that Digital HR journey too has its own challenges. It requires a considerable bandwidth of team members and has lot of potential to impact the organization's overall productivity. As per report of the World Economic Forum, diversified workplaces would emerge in the industry due to mobile, cloud technology, big data and computing power. In another two years, it predicts, that AI (Artificial Intelligence), ML (Machine Learning), and advanced robotics drive the workforce increasingly.

How much will the Digitalization Impact HR?

The digitalization transforms the way things are done. Everything seems to be self-driven and automated. None of the companies would prefer to be out of the digital race and remain outdated, and almost every company is investing in making whatever they do to be digitalized. The question, thus, arise - will the digitalization make the HRM function an obsolete? This study reveals interesting facts in this regard. 


\section{- Process Acceleration:}

Digitalization tools such as Artificial Intelligence will cut short the laborious processes. For example, to fill up the hundreds of job vacancies if it takes a week's time for the HR to manually shortlist and evaluate them, the AI takes only a few minutes. It searches the database with the keywords such as location, technical skills, number of years of experience, educational qualifications, and specializations, if any, and so on. (Reference: Financial express). It can also send automated emails to the shortlisted candidates and schedule interviews. In fact, it is reported that "Monjin" kind of video-interviewing tools helped improving hiring accuracy by almost $86 \%$.

\section{- Increase Transparency:}

If we look at an emerging technology called as Block chain, also called as Distributed Ledger Technology (DLT), it is a database that maintains the complete list of digital records, which are increasing continuously day by day with no end in sight. In HR, it is perceived that digital process management can be managed to increase transparency. It also helps automate overseas electronic payroll. The DLT addresses the certification issues, simplifies the dataheavy processes ensuring the cybersecurity and fraud prevention.

\section{- Collaborate and Connect with Ease:}

In a Webinar on the topic of "why do collaboration-tools matter for a disbursed workforce", Mr. Suresh Mani, Head HR Ops and Shared Services quoted that Aditya Birla, with an employee size of 14000 with over 600 branches across the country, leveraged conversational interfaces that helped in increased responsiveness within the company/workforce, no matter wherever they are, and all service requests or transactional queries are attended to within no time. Prior to this, it was observed that $50 \%$ of employees queries remain unanswered even after 24 hours. $30 \%$ of an employee's tasks often get delayed due to dependency on others. $20 \%$ of an employee's productive time is spent in coordinating or managing work-life related transactions or service requests.

Thus, conversational interfaces or conversational tools such as Chat bots, Messaging Apps and Intelligent Assistants, which are available to employees $24 \times 7$, foster workplace productivity and team work by reducing the time that they spend on transactional queries.

\section{- Helps Identifying the Resources with Better Fitment:}

In talent acquisition, many recruiters use big data analytics to match as much selection criteria as possible with the profiles of the candidates, based on the job description to hire the right candidate. This will also help the candidate to meet his/her expectations if got selected, and thereby the tenure would be longer in the organization, and the retention period is high. As big data generates and provides valuable insights of the candidates, the HR/hiring manager will be able to take appropriate hiring decisions.

\section{- Work-Life Balance:}

Let us look at a growing new community by name Digital nomads in this era. These are the people who are "location independent" and use technology (internet) to perform their job. They are professionals who prefer to work remotely, primarily to enjoy their freedom. They may be writers, techies, marketing professionals, creative designers, HRs etc. Digital nomads might be insensitive to their routine office timings, but are very sensitive about their deliverables and timelines. These are often less-stressed and are more productive than those who work in offices, and hence they maintain great work-life balance.

\section{Objectives of the Study:}

The study is undertaken to achieve the following objectives:

(i) To understand how the digitalization in HRM is emerging.

(ii) To draw attention to the impact on the HRM.

(iii) To gain insight on how effective is the digitalization in HRM.

\section{Research Methodology}

The study is conceptual in nature. Hence, secondary source of data mostly comprising journals, research publications, and internet has been used to achieve the stated objectives.

\section{Digitalization and its Effectiveness on HRM \\ - Diverse Workforce:}

The contemporary workforce is diverse and distributed as they join us from different geographical locations having different cultural backgrounds and mindsets. The digitalization and the technological tools are to be designed in such a way that it suits the people from all walks of life. For example attendance tracking based on the time zones, observation of holidays depending on the customs prevailed in the local region, engaging the workforce with the communications that suit to their nativity. 


\section{- Consumer Protection and Regulatory Compliant:}

While digitalizing, the organizations must keep in view the protection of customer sensitive information, financial integrity concerns, speed of transactions, environmental footprint, legal, regulatory and technological issues that arise with the advent of new technology. If they tend to compromise on anyone of these, it will have an appalling impact on the systems and processes in the organization, and there could be a possibility of tarnishing the image of the organization, which could be irreversible. The organizations should maintain utmost data privacy and data integrity for the reason everything is stored in databases and the access should be restricted only to the authorized members and to the extent it is necessary failing which the confidential information will be leaked out.

- Not Everything can be left to Automation:

Everything being said and done, it is believed that everything cannot be left to automation. While an HR can always bank on the artificial intelligence system to ask questions during interviews, but they can't depend on it to analyze candidates' personality, their zeal for the job, or their soft skills. The digitalized systems can't negotiate with a candidate nor the tools cannot solely on-board an employee nor create a great candidate experience, because systems lack a quality that the humans are blessed with - the power of intuition. HR is a human-centric function. While the technology can help with other aspects such as automating mundane work and saving time, it cannot replace the human quotient involved.

\section{- Understanding the Digitalization in the Right Perspective:}

No one can deny the fact that the organizations should quickly adopt to the latest technological advancements, go with the flow, and make use of its benefits. But, while people focus on the number of jobs lost to machines on account of digitalization, many fail to see the flipside that the new jobs that are created for them. New roles such as that of an AI Trainer, Machine Learning Engineer and AI Interaction Manager, which are unheard of earlier. According to a study by NYT, approximately only 10,000 people worldwide are qualified for the forthcoming roles. Most of the companies reported to have failed ( $75 \%$ of all ERP implementations) in the implementation of digitalization, as per the study by Gartner. The reason is said to have been that the companies do not have defined goals and specific objectives. They failed to identify probable timelines.

\section{Conclusion}

Hence, the question here is not whether AI, ML or automation replace the HR. The question is all about how well the resources including HR people are well equipped by learning and getting acquainted with these newer technologies. It is the question of how can AI be used by humans to improve their job. When the organizations document the current processes, following by creating awareness among employees to give them hands-on exposure on the digitalized tools, the exercise would help build the confidence in the system. The digitalization in HRM has to be integrated with the overall HR strategies and policies thereby giving access to employees empowering them in all aspects to ensure greater transparency. Then only every employee will be fully informed and self-sufficient.

\section{References}

1. https://www.peoplematters.in/article/technology/how-the-worlds-largest-cosmetic-company-transformed-itshiring-practice-with-ai-19006

2. https://www.financialexpress.com/jobs/hr-jobs-will-ai-retire-recruiters/1227482/

3. https://www.gartner.com/en/newsroom/press-releases/2016-12-21-gartner-says-by-2019-20-percent-of-userinteractions-with-smartphones-will-take-place-via-vpas

4. http://www.hrkatha.com/news/2490-your-digital-footprints-can-make-or-break-your-career

5. https://pm1-31ef.kxcdn.com/docs/hipo_survey_report.pdf

6. http://www.hrkatha.com/news/2483-the-growing-community-of-digital-nomads-in-the-workforce-PrajjalSaha HR Katha | New Delhi | Friday, 10 August 2018)

7. https://www.worldbank.org/en/topic/financialsector/brief/blockchain-dlt) 